\title{
Enabling Practical Backscatter Communication for On-body Sensors
}

\author{
Pengyu Zhang, Mohammad Rostami, Pan Hu, Deepak Ganesan \\ College of Information and Computer Sciences \\ University of Massachusetts, Amherst, MA 01003 \\ \{pyzhang, mrostami, panhu, dganesan\}@cs.umass.edu
}

\begin{abstract}
In this paper, we look at making backscatter practical for ultra-low power on-body sensors by leveraging radios on existing smartphones and wearables (e.g. WiFi and Bluetooth). The difficulty lies in the fact that in order to extract the weak backscattered signal, the system needs to deal with self interference from the wireless carrier (WiFi or Bluetooth) without relying on built-in capability to cancel or reject the carrier interference.

Frequency-shifted backscatter (or FS-Backscatter) is based on a novel idea - the backscatter tag shifts the carrier signal to an adjacent non-overlapping frequency band (i.e. adjacent WiFi or Bluetooth band) and isolates the spectrum of the backscattered signal from the spectrum of the primary signal to enable more robust decoding. We show that this enables communication of up to 4.8 meters using commercial WiFi and Bluetooth radios as the carrier generator and receiver. We also show that we can support a range of bitrates using packet-level and bit-level decoding methods. We build on this idea and show that we can also leverage multiple radios typically present on mobile and wearable devices to construct multi-carrier or multi-receiver scenarios to improve robustness. Finally, we also address the problem of designing an ultra-low power tag that can frequency shift by $20 \mathrm{MHz}$ while consuming tens of micro-watts. Our results show that FS-Backscatter is practical in typical mobile and static on-body sensing scenarios while only using commodity radios and antennas.
\end{abstract}

\section{CCS Concepts}

$\bullet$ Networks $\rightarrow$ Network architectures; Wireless access networks;

Permission to make digital or hard copies of all or part of this work for personal or classroom use is granted without fee provided that copies are not made or distributed for profit or commercial advantage and that copies bear this notice and the full citation on the first page. Copyrights for components of this work owned by others than ACM must be honored. Abstracting with credit is permitted. To copy otherwise, or republish, to post on servers or to redistribute to lists, requires prior specific permission and/or a fee. Request permissions from permissions@acm.org.

SIGCOMM '16, August 22-26, 2016, Florianopolis , Brazil

(C) 2016 ACM. ISBN 978-1-4503-4193-6/16/08 . . \$15.00

DOI: http://dx.doi.org/10.1145/2934872.2934901

\section{Keywords}

Backscatter; Sensor; Wireless

\section{INTRODUCTION}

The ultra low-power nature of backscatter communication makes it a compelling technology for the design of wearable and on-body sensors that operate on tiny energy budgets. Today, most such sensors use Bluetooth Low Energy (BLE) for low-power communication, but BLE consumes tens of milliwatts when operating in active mode i.e. when transmitting data. In contrast, a backscatter tag consumes a few micro-watts in active mode, and enables the design of onbody sensors that continually stream data at an end-to-end power budget of tens of micro-watts [56]. The tiny energy budget combined with the simplicity of the hardware components needed to design backscatter-based sensors opens up a range of possibilities including micro-powered on-body sensors [52], miniature implantable sensors [51], thin and flexible wearables [39], and others.

But when we attempt to make backscatter practical for on-body sensors, we face a conundrum. Unlike built environments where backscatter-enabled access points or readers can conceivably be deployed, we have limited options in a mobile environment. We can perhaps modify radio chipsets in smartphones and wearables to include backscatter support, but this will not be immediately deployable and their widespread use will hinge on market forces. Ideally, we would leverage existing mobile and wearable devices that people already use as a source of continuous carrier and backscatter receiver. But these devices are not designed to support backscatter, and therefore do not embed crucial building blocks such as self-interference cancelation. This is particularly problematic when dealing with a link as fickle and sensitive as backscatter — reverse link path loss and backscatter antenna reflection losses create a dicey decoding scenario even with perfectly tuned hardware [45], and the constraints of commercial transceivers on mobile devices only exacerbates the situation.

Consider the case of WiFi Backscatter [30], a recent attempt at resolving this conundrum. In this technique, a receiving WiFi device looks at the RSSI or CSI values of each packet, and first smoothes these values to remove natural variations in the WiFi signal. It then uses signal strength 
variations in the averaged signal to extract a lower rate backscattered signal. This approach works in an Internet of Things context when a static backscatter tag can be equipped with a large antenna. However, it is difficult to tune precisely in a mobile scenario where a mobile tag is equipped with a small antenna and more importantly, the WiFi signal is continuously changing due to movement and body blockage variations. This makes it hard to cleanly average away the WiFi signal variations, and leads to low signal to noise ratio (SNR), and consequently less performance in terms of range and throughput. Thus, the challenge that we face is how to use commercial transceivers while also effectively dealing with carrier interference.

Our key insight in this paper is that backscatter can be made practical for wearables using a simple but effective trick - if a backscatter tag can shift an incident WiFi or Bluetooth carrier to a clean WiFi or Bluetooth band, then that the receiver can see a clean, carrier-interference free backscattered signal in the shifted band. The tag can perform on-off keying (OOK) at the shifted frequency to transfer information in the shifted frequency band. This method is practical on devices that many users already use in mobile settings. For example, a mobile phone can act as a Bluetooth carrier, an on-body sensor can be a tag that shifts the signal by $20 \mathrm{MHz}$ while modulating it, and a Bluetooth receiver on a wristband (like a Microsoft Band) can receive this shifted signal in the adjacent band.

There are two reasons why frequency shifting allows us to improve backscatter performance. The first is that the receiver sees a clean signal and does not need to deal with any other interference in the same channel. The lower noise level means that we can achieve higher performance than methods that try to separate the primary carrier from backscatter signal in a single channel without assistance of self-interference cancelation techniques. The second reason is that the receiver can use the structure of the primary carrier (i.e. WiFi or Bluetooth packet preamble) to be able to detect the shifted signal at very low SNRs. For example, typical WiFi and Bluetooth chipsets have receive sensitivity of $-90 \mathrm{dBm}$ to $95 \mathrm{dBm}$, much lower than the threshold of detecting the RSSI of a signal with unknown structure. This allows us to operate at longer ranges than RSSI-based methods, albeit at lower bitrates since we can modulate information only at the rate at which packets are transmitted. Thus, our method leverages both the benefits of frequency shifting as well as the high receive sensitivity of modern radio chipsets.

Frequency shifting also opens up some interesting new possibilities. We often have multiple portable devices in our vicinity including phones, smartwatches, tablets and laptops. In these scenarios, we can leverage multiple transmitters and receivers to improve the throughput and reliability of the link. This is possible since the tag simply reflects any incident signal that resonates with its antenna unlike active radios that need to filter signals into specific bands before transmission.

While frequency shifting has many benefits, it opens up a fundamental challenge of tag-side power consumption. Shifting to an adjacent $\mathrm{WiFi}$ band necessitates a $20 \mathrm{MHz}$ oscil- lator at the tag, whereas existing RFIDs and computational RFID-scale devices only need slow oscillators that operate at several Kilohertz. High speed oscillators typically consume milliwatts of power, which is incompatible with our goal of operating at micro-watts of power. We tackle this challenge by sacrificing precision for power - we design a low-power ring oscillator-based clock generator for the FSBackscatter tag which operates at tens of micro-watts but also has temperature-induced frequency variations. However, we show that FS-Backscatter is robust to such temperature induced frequency variations that we might expect for on-body sensors.

In summary, our system, FS-Backscatter, has several novel contributions.

- First, we design, implement and evaluate a practical backscatter system for on-body devices that enables ultra-low power communication while also being compatible with commercial WiFi and Bluetooth transceivers. We show that FS-Backscatter can operate up to $4.8 \mathrm{~m}$ distance and provide throughputs ranging from tens of bits/second to tens of kilobits/second depending on the specific transmitter - receiver configuration.

- Second, we show that FS-Backscatter can take advantage of the plethora of radios that are available on portable devices and combine transmitters or receivers to boost performance. We show that throughputs increases by $25 \%$ to $100 \%$, and we can achieve up to $48.7 \mathrm{kbps}$ throu- ghput in two transmitter and two receiver scenarios.

- Third, we show that an FS-Backscatter tag operates at a power budget of $45 \mu \mathrm{W}$ through the use of a ringoscillator based clock design, and is robust to frequency variations induced by environmental changes.

\section{CASE FOR FS-BACKSCATTER}

Several recent efforts have proposed ways to make backscatter communication practical by leveraging either existing wireless infrastructure or existing wireless-enabled devices. The mobile scenario, which is the target of our work, adds an additional wrinkle in that the method should work on-thego and not just in built settings. We discuss prior work from this perspective and understand how they fare in our problem domain.

\subsection{Infrastructure-assisted Backscatter}

Several existing techniques rely on tethered infrastructure either for carrier generation or for decoding the backscattered signal or both. Of course, all RFID readers operate in this manner in that they generate a narrowband carrier, and perform self-interference cancelation to separate the backscattered signal from the carrier. But RFID reader infrastructure is not ubiquitous, so a few recent methods have designed innovative ways to embed reader functionality into existing devices.

BackFi [9] modifies a WiFi Access Point (AP) by augmenting it with the ability to cancel the OFDM carrier signal. The benefit of this technique is that it keeps the tag very sim- 
ple - a simple ASK-transmitting tag can simply backscatter the AP-generated WiFi signal without worrying about the complexity of the underlying OFDM signal structure.

BLE-Backscatter [13] flips this method and provides infrastructural support such that a backscatter tag can communicate with a commodity BLE radio receiver. Here, the infrastructure component is a simple continuous wave $(\mathrm{CW})$ transmitter, and a backscatter tag modulates the $\mathrm{CW}$ tone to emulate a BLE transmitter, thereby allowing commodity BLE receivers to receive the modulated signal. The BLEBackscatter tag saves power because it no longer needs to generate the carrier, but it emulates a BLE stack and is therefore more complex and power-hungry than an ASK-modulating backscatter tag.

Passive WiFi [31] requires similar infrastructure support as BLE-Backscatter and enables backscatter communication between a tag and a commodity $802.11 \mathrm{~b} \mathrm{WiFi} \mathrm{device.} \mathrm{Sim-}$ ilar to the BLE-Backscatter case, Passive WiFi includes a carrier emitter, which transmits a constant single tone signal. A Passive WiFi tag generates and modulates an $802.11 \mathrm{~b}$ baseband signal on the emitted single tone signal during reflection. With the help of the single tone emitter, Passive WiFi enables the backscatter communication between a tag and a commodity $802.11 \mathrm{~b}$ WiFi radio without any hardware modification on the WiFi device. However, a Passive WiFi tag needs to generate the entire $802.11 \mathrm{~b}$ baseband signal, and therefore, is more complex and power hungry compared to an ASK-modulating backscatter tag.

Neither of these methods are viable in a mobile context since they use infrastructure-assistance, and require additional hardware for self-interference cancelation or $\mathrm{CW}$ generation that is not embedded in existing radios.

\subsection{Infrastructure-less Backscatter}

A second class of methods leverages an ambient carrier (e.g. TV or WiFi carrier), and backscatter this signal so that it can be received at a commodity receiver. Of these, we do not consider the TV carrier signal used by Ambient Backscatter [34] since its availability is spotty and its signal strength decays a few miles away from a TV tower station. So, this technique is less appropriate for continuous monitoring in a mobile scenario. But WiFi Backscatter [30] could be practical since it uses a commodity WiFi transmitter and receiver, which is plausible in a wearable scenario where we might use a phone as the transmitter and smartwatch as receiver. The tag side retains the simplicity of ASK-based backscatter.

From a signal processing perspective, the key challenge is separating the ambient carrier from the backscattered information without the benefit of self-interference cancelation. Instead, these methods rely on the fact that changes in the WiFi or TV carrier occur at a much higher rate than changes in the backscatter modulation. Therefore if the received signal is averaged over a long enough window, the backscatter modulated information can be recovered. This averaging can be done using an envelope detector in the analog domain (used in Ambient Backscatter [34]), or low pass filter in the digital domain (used in WiFi Backscatter [30]), after

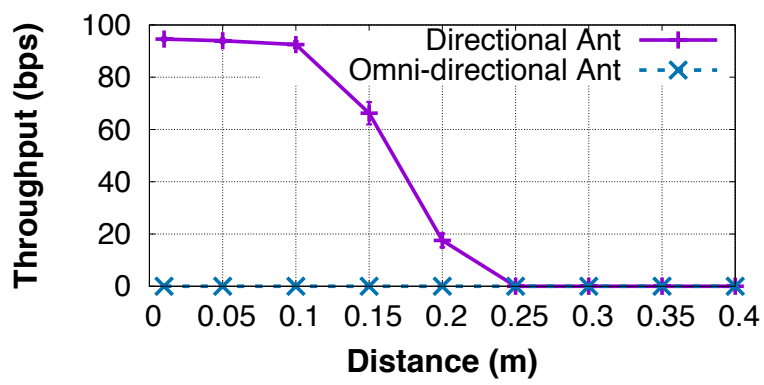

Figure 1: Throughput of WiFi Backscatter across distance with 3dBi Omni-directional [2] and 9dBi directional [5] antennas.

which one can measure how a backscattered signal changes the propagation characteristics of the incident signal.

While WiFi Backscatter was shown to work in a static IoT setting, there are some key challenges in using this technique in a wearable scenario. The design presents two issues: a) the primary exciter is much louder than the backscatter signal and, despite averaging, reduces signal to noise ratio to such an extent that range is extremely low, and b) the temporal variations due to typical human movements and corresponding channel variations in mobile environments requires dynamic tracking of signal and noise thresholds, which in turn makes decoding sensitive to the chosen thresholds.

To understand its performance, we empirically measure WiFi Backscatter throughput across distance. We use a bistatic backscatter deployment similar to that in [30], and place a CC3200 WiFi transmitter 1m away from a backscatter tag while moving the CC3200 WiFi Backscatter decoder away. The results are shown in Figure 1 .

Our first observation is that when a tag is equipped with a standard 3dBi omni-directional antenna [2], we do not observe RSSI variations introduced by WiFi Backscatter. Therefore, zero backscatter throughput is achieved. We then try to equip the tag with a 9dBi directional antenna [5] and find that WiFi Backscatter works up to $0.2 \mathrm{~m}$ and achieves $19 \mathrm{bps}$ data rate. Let us try to understand why WiFi Backscatter has low performance.

Low signal-to-noise ratio The first key issue is the strong interference from the ambient carrier, which limits operational range as well as data rate. To measure interference, we set up a deployment similar to the one in [30], and place a OdBm WiFi transmitter $3 \mathrm{~m}$ away from a backscatter device. One difference is that our tag is equipped with an omnidirectional antenna, unlike [30] which uses a custom multiantenna array. The main reason for this change is that the $18.5 \mathrm{~cm} \times 15.7 \mathrm{~cm}$ custom multi-antenna array is too large for on-body sensor tags. We move the WiFi receiver away from the backscatter tag and measure the TX signal strength as well as the backscattered signal strength.

Figure 2 shows empirically measured SNR and SINR of WiFi Backscatter across distance. Even when the receiver is $0.1 \mathrm{~m}$ from the backscatter device, the SINR measured is -

\footnotetext{
${ }^{1}$ We can measure the antenna size using the picture shown in [30] because the size of each patch element is $4.06 \mathrm{~cm} \times 3.09 \mathrm{~cm}$.
} 


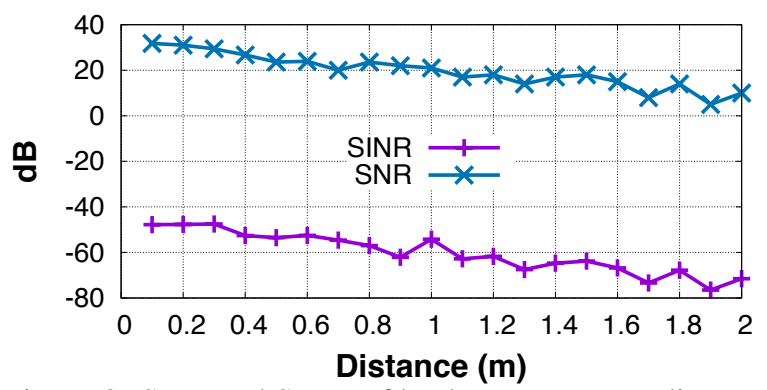

Figure 2: SNR and SINR of backscatter across distance.

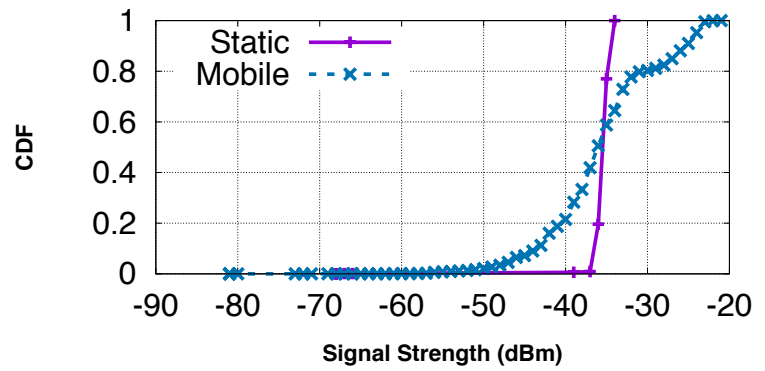

Figure 3: Received WiFi signal strength in static and mobile deployment.

$47 \mathrm{~dB}$ i.e. the transmitted WiFi signal strength is $47 \mathrm{~dB}$ higher than the backscattered signal strength. When the receiver is moved further, the SINR decreases even more. The SINR at $2 \mathrm{~m}$ decreases to $-71 \mathrm{~dB}$, which makes backscatter decoding challenging. As a result, the system can achieve respectable data rates only at short ranges of a few centimeters, and decoding range is typically a meter or less while the data rate is reduced to a few bits/second.

Mobility-induced dynamics The second issue is that mobility changes the propagation characteristics of an incident signal, which makes decoding highly sensitive to the chosen threshold. Figure 3 shows the CDF of the received signal strength of a WiFi transmitter over 10 minutes when it is placed $1 \mathrm{~m}$ away from a receiver. When the transmitter and receiver are static, the environment does not change and we can observe a stable WiFi signal with a median strength of $35 \mathrm{dBm}$. However, when a person carries both the transmitter and receiver and moves around, the received signal strength varies significantly from $-80 \mathrm{dBm}$ to $-20 \mathrm{dBm}$. Such dramatic signal variations will introduce significant decoding errors if the pre-calibrated threshold is not adapted accordingly. But adaptive re-calibration of the threshold is also very hard due to the large dynamic range of the variability, and will require complicated channel estimation and adaptation that is well outside the regime of what can be done on an ultra-low power backscatter tag.

\subsection{FS-Backscatter: Key Ideas and Chal- lenges}

The essential idea underlying FS-Backscatter is quite simple - if a tag can shift-and-modulate a carrier signal in one frequency band into an adjacent non-overlapping frequency band where a receiver is listening, then the receiver has a clean channel within which to recover the modulated

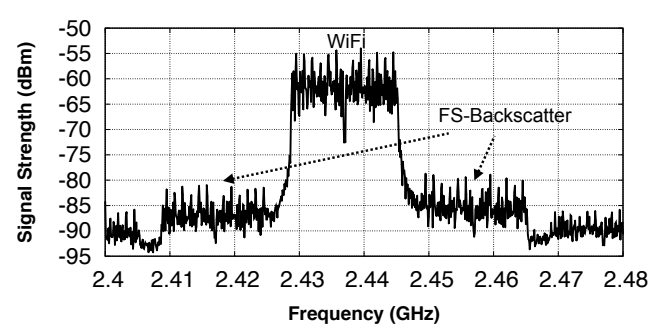

(a) FS-Backscatter reflects a WiFi signal

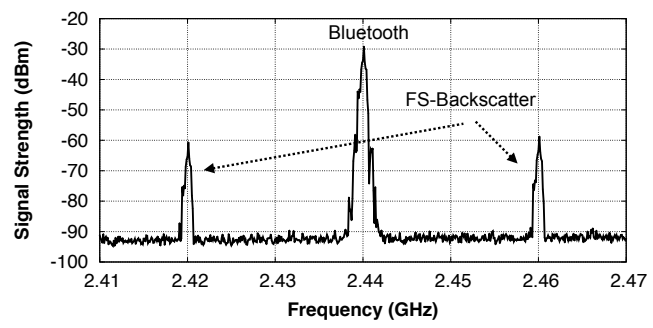

(b) FS-Backscatter reflects a Bluetooth signal

Figure 4: FS-Backscatter reflects a WiFi signal and a Bluetooth signal to adjacent non-overlapping channels.

backscatter signal. This model is quite different from existing methods for backscattering which either use ASK or FSK modulation; instead, our method involves a fixed frequency shift to a clean band followed by amplitude modulation.

Why would we expect this method to work well? The first reason is simply that the backscattered signal is shifted into a clean band where we are no longer affected by the interference from the carrier. Figure 4(a) shows the effect of shifting a WiFi signal, and Figure 4(b) shows the same result for a BLE signal. It is clear that the shifted signal is quite distinct from the primary carrier.

A second reason is that modern WiFi and Bluetooth receivers are designed to be extremely sensitive to structured weak signals, such as the preamble in a packet. For example, the CC2560/CC2564 Bluetooth receivers are able to detect packets at $-95 \mathrm{dBm}$ which allows them to work at a few tens of meters while only consuming tens of milliwatts. We can leverage this sensitivity to combat signal losses due to reflection (typically $30 \mathrm{~dB}$ ) and due to path loss on the reverse link. The distance we need to operate under in typical mobile scenarios is only a couple of meters, which is much shorter than the receive range of either Bluetooth or $\mathrm{WiFi}$ and gives us room to have additional signal losses due to body attenuation.

While frequency shifting opens up an array of possibilities, it introduces some practical questions and challenges. The first question is one of practicality - is this technique viable in practice? If it is viable, how well does it perform? When does it work and when does it fail? Do commodity radios expose APIs that allow us to tap into this method? The second is one of power - since non-overlapping $\mathrm{WiFi}$ bands are separated by $20 \mathrm{MHz}$, we need a $20 \mathrm{MHz}$ oscillator at the tag. This is substantially higher than what is needed for simple ASK modulation at a few tens or hundreds of kilobits/second, and higher frequency clocks incur more power. 


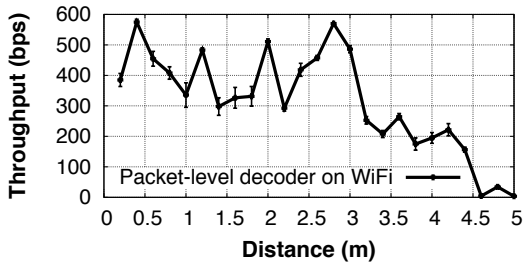

(a) Packet-level decoding on reflected WiFi.

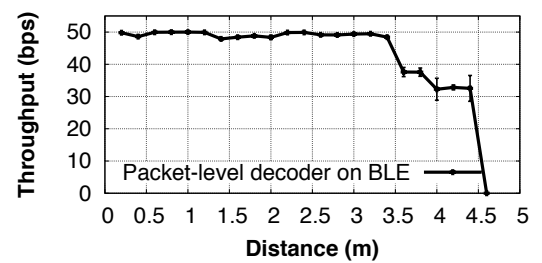

(b) Packet-level decoding on reflected BLE.

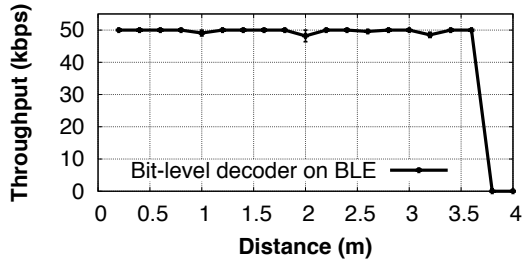

(c) Bit-level decoding on reflected BLE.

Figure 5: FS-Backscatter throughput across distance when leveraging WiFi and Bluetooth signals.

But how much power efficiency do we lose at the tag? Are there ways to mitigate the loss of efficiency and keep it to tens of micro-watts? In the rest of this section, we discuss answers to these questions.

\section{FREQUENCY-SHIFTED BACKSCAT- TER}

In this section, we look at the practicality of FS-Backscatter on existing commodity radios and the implications on the design of the tag. We start with single transmitter to receiver scenarios, then at multiple transmitters to receivers scenarios, and finally discuss the design of the tag.

\subsection{FS-Backscatter on Commodity Radios}

The first question we ask is: If we take a commodity WiFi or Bluetooth Low Energy (BLE) chipset operating in broadcast mode, and shift the carrier to the adjacent frequency band while simultaneously modulating the carrier in this band, can a receiver listening on the adjacent band decode the backscattered signal?

\subsubsection{Packet-level FS-Backscatter}

Our first set of experiments look at the packet-level RSSI information that most $\mathrm{WiFi}$ and BLE chipsets provide, and see whether this can be used to decode the backscattered signal.

WiFi-to-WiFi Backscatter In this experiment, a CC3200 WiFi transmitter transmits a stream of packets in channel 1, and a WiFi receiver (CC3200) is configured to listen to packets in the next non-overlapping channel 5. The transmitter transmits at 1200 packets/second, and a FS-Backscatter tag is configured to shift by $20 \mathrm{MHz}$ and then perform on-off keying of its RF transistor at half the frequency of the transmitter packet rate i.e. 600 bits/second. The idea is that the WiFi receiver successfully receives a packet when the tag shifts by $20 \mathrm{MHz}$, and does not receive a packet when the tag does not shift. This binary sequence of bits is the information being transmitted by the backscatter tag.

Figure 5(a) shows the results when the WiFi transmitter is $1 \mathrm{~m}$ away from the tag and we move the receiver away from the tag. The frequency shifted signal can clearly be decoded by the receiver. FS-Backscatter is able to operate up to $4.8 \mathrm{~m}$ when it leverages packet-level RSSI information for decoding and has average throughput of $313.8 \mathrm{bps}$ across all distances.

Bluetooth-to-Bluetooth Backscatter The same underlying method for frequency shifting can also be used with a TI
CC2650 BLE transmitter and a BLE receiver listening on the channel that is $20 \mathrm{MHz}$ away. The transmitter broadcasts at 100 packets/second. Figure 5(b) shows that FS-Backscatter is able to operate up to $4.4 \mathrm{~m}$, with an average data rate of 45.8bps.

\subsubsection{Bit-level FS-Backscatter}

The above approach shows feasibility, but throughput is quite low since we are limited to one piece of information (RSSI) per packet. This means that any backscatter modulation scheme is limited by the packet rate on commodity radios - WiFi can broadcast about $3 \mathrm{~K}$ packets/second, while BLE only broadcasts $\sim 100$ packets/second. These rates are comparable to what was achieved in WiFi Backscatter, but given that we have a clean band to work with, we should be able to go a lot faster. But to achieve this, we need information at a layer lower than packet-level RSSI i.e. we need sub-packet RSSI information.

To explore this option, we use a commercial TI BLE radio that exposes a slightly lower level interface [4]. This radio provides an option for bypassing the BLE stack and directly obtaining RSSI values of the channel at a finer granularity. This physical layer interface can be used for detecting the presence (or absence) of a backscattered signal in the band at rates that are considerably faster than packet-level backscatter.

In this experiment, we use a Bluetooth transmitter, and configure an FS-Backscatter tag to modulate at a rate of 50kbps. We sample RSSI information at $100 \mathrm{KHz}$ from the CC2541 $\mathrm{BLE}$ receiver to decode the signal. Thus, each RSSI reading is an average of the channel readings over a duration of $10 \mu \mathrm{s}$, and provides a measure of whether or not the backscattered signal is present in the adjacent channel.

Figure 5(c) shows the results. We can see that FS-Backscatter is able to achieve $\sim 50 \mathrm{kbps}$ data rate at close range and can operate up to $3.6 \mathrm{~m}$. The range is shorter than packet-level backscatter since we are not able to exploit structure in the backscattered signal that is used for packet-level decoding. But we are able to take advantage of the fact that we are working in a clean channel with limited noise, and thereby operate over a longer distance than techniques that use ASK backscatter without frequency shifting.

\subsubsection{What if no channels are available?}

In the previous discussion, we assumed that the channel adjacent to the carrier is unoccupied, but one question is what if none of the channels are free. Our backscattering method 


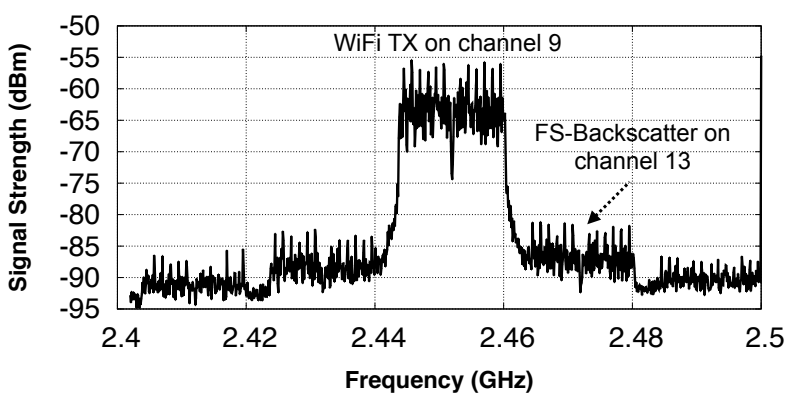

Figure 6: FS-Backscatter spectrum when leveraging $2.4 \mathrm{GHz}$ WiFi channel 13 for carrying backscattered information.

works only when two adjacent non-overlapping channels are available i.e. the transmitter channel, and either the channel at the next lower non-overlapping frequency band or the higher non-overlapping frequency band. Note that both are viable options since frequency shifting shifts the carrier into both adjacent channels. But it is not unusual for many wireless channels to be occupied, so what happens if that is the case.

We note that even if there is a significant amount of WiFi traffic, some channels are highly unlikely to be used for active transmission. $2.4 \mathrm{GHz} \mathrm{WiFi}$ has 14 allocated channels, whereas only 11 are used in practice since channels 12 and 13 have strict requirements regarding emission limits to avoid spilling over to adjacent restricted frequency bands [12]. However, since the backscattered signal is very weak, it is well below these emission limits, and hence we can shift the carrier from Channel 9 and listen in Channel 13.

We verify the emissions from FS-Backscatter in Channel 12 and 13 when a WiFi transmitter is operating in Channel 9. Figure 6 shows that the backscattered signal strength at Channel 13 is only $-85 \mathrm{dBm}^{2}, 30 \mathrm{~dB}$ lower than the WiFi carrier signal and close to the noise level. Therefore, FSBackscatter will not cause interference to radios operating close to Channel 13 because its signal strength is too weak.

\subsubsection{Can we improve robustness by using mul- tiple transmitters or receivers?}

So far, we have discussed the case where there is a single incident carrier and a single receiver. But in many mobile scenarios, we have the possibility of using more than two radios. For example, we often have multiple bluetooth-enabled accessories including tablets and headsets, so we may be able to repurpose these as an additional backscatter carrier or receiver. These additional radios can potentially be used as multiple carrier emitters and receivers to improve robustness since backscattered signals are generally weak and more sensitive to noise.

Can FS-Backscatter leverage more than two radios? One of the benefits of FS-Backscatter is that it is not limited to backscattering a single carrier. The backscatter tag's ana$\log$ RF front end includes only an RF transistor and antenna, and unlike other radios, has no filters to limit the band where the radio can operate. As a result, a backscatter tag is able to reflect multiple incident signals at the same time as long

\footnotetext{
${ }^{2}$ Measured at the FS-Backscatter tag antenna.
}

as these signals can resonate with the backscatter antenna. Since both WiFi, Bluetooth, Zigbee and many other ISMband radios share the same $2.4-2.483 \mathrm{GHz}$ spectrum, a backscatter device is able to reflect some combination of these at the same time. This feature provides several potential benefits where we can leverage multiple ambient carriers and multiple receivers to enhance backscatter performance.

We can leverage multiple transmitters and receivers quite easily in FS-Backscatter. Multiple transmitters can simply turn off carrier sensing and broadcast in the same band to increase the reflected signal strength. Note that this method would not work if we use ASK backscatter in the same channel as the carrier, since the additional transmitter would also add interference. But in FS-Backscatter, the backscatter signal strength is boosted in the shifted channel.

If we use multiple receivers, we can simply combine the signals to improve decoding performance. In an ideal scenario, one could combine the analog signals via maximal ratio combining, but since we operate over a commercial transceiver, we are restricted to the RSSI information coming from the radio. Thus, in our case, the two receivers can measure the signal strength (RSSI) of a backscattered bit on each receiver, and exchange this information. Then, we simply choose the bit that is received with higher RSSI for determining the actual bit transmitted by a backscatter tag.

\subsection{Low-power FS-Backscatter Tag}

A major question that remains is the design of the FSBackscatter tag. The main consideration is that the tag needs to be able to shift by $20 \mathrm{MHz}$ such that it can shift both WiFi and Bluetooth carriers into a non-overlapping frequency band. This is a key difference between an FS-Backscatter tag and previous work on RFIDs (and Computational RFIDs) since previous work focuses either on ASK or FSK modulation around the center frequency of the carrier, whereas we require the the tag to shift the carrier by $20 \mathrm{MHz}$ prior to modulation. Thus, the question we need to answer is whether an FS-Backscatter tag can operate at micro-watts of power while shifting the carrier by such a substantial amount.

\subsubsection{What is the power bottleneck?}

Intuitively, more power will be consumed when we have to shift the carrier by larger frequencies. We look at three subsystems on a backscatter tag - RF transistor, transmission logic and clock generator, to determine which of these consume the most power as the shifted frequency increases.

RF Transistor The RF transistor is a MOSFET transistor with a capacitance around $2.1 \mathrm{pF}$ (ADG902). Its power consumption can be calculated using the equation $\frac{1}{2} C V^{2} F$ where $C$ is the capacitance of the transistor, $V$ is the gate voltage, and $F$ is the frequency of operating the transistor. Even when toggled at a high rate of $20 \mathrm{MHz}$, the RF transistor only consumes $21 \mu \mathrm{W}$. Thus, the power consumption of the RF transistor itself is low and has a linear relationship with $F$.

Transmission logic The second subsystem, transmission logic, is a hardware module that toggles the backscatter RF transistor based on data transmitted. We use a digital cir- 
Table 1: Power consumed by commercial oscillators operating at different frequencies and different accuracies.

\begin{tabular}{|c|c|c|c|}
\hline Oscillators & Frequency & Accuracpy & Power \\
\hline ASH7K & $32 \mathrm{kHz}$ & $\pm 10 \mathrm{ppm}$ & $1.48 \mu \mathrm{W}$ \\
\hline LTC6990 & $1 \mathrm{MHz}$ & $\pm 50 \mathrm{ppm}$ & $326 \mu \mathrm{W}$ \\
\hline LTC6900 & $10 \mathrm{MHz}$ & $\pm 40 \mathrm{ppm}$ & $2.04 \mathrm{~mW}$ \\
\hline
\end{tabular}

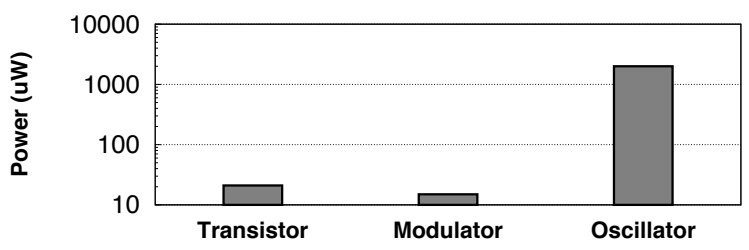

Figure 7: Backscatter tag power consumption breakdown.

cuit to implement the transmission logic, and the power consumption of this module increases linearly with the rate of transmission [56]. While the precise power consumption depends on the logic, we expect that this module consumes around $15 \mu \mathrm{W}$ of power given that we can open and close the transistor via an NAND gate [3], which has a capacitance of around $1.5 \mathrm{pF}$.

Clock generator The third subsystem is the clock generator which provides the clock for timing the whole system. Oscillators are typical sources for generating clocks. Table 1 shows the power consumed by the lowest power commercially available oscillators that we could find at different frequencies and accuracies. We find that once we begin shifting by several $\mathrm{MHz}$, the power consumption also rises to a few milliwatts.

Figure 7 shows a power consumption breakdown of the three subsystems. Its clear from the above breakdown that the clock generator is the highest power consumer in the entire system and consumes two orders of magnitude of more power compared to the RF transistor and the transmission logic. So we turn our attention to this component and ask whether there is a way to make our oscillator circuit operate at $\mu$ Ws of power.

\subsubsection{Can we shift by $20 \mathrm{MHz}$ while consuming $\mu W s$ ?}

A key question in designing a low-power oscillator is the precision that we are willing to tolerate. Active radios choose their oscillators based on several considerations including reducing leakage outside the channel to permitted levels, lowering phase noise, and minimizing power consumption. But if FS-Backscatter can tolerate less precision in the oscillator output, we can design significantly lower power oscillators.

In particular, one attractive design for an ultra-low power oscillator is a ring oscillator, which is used in some integrated digital and communication systems [28] [46] [32]. Our design of a $20 \mathrm{MHz}$ ring oscillator looks as shown in Figure 8 A ring oscillator leverages an odd number of inverters and connects them in a serial sequence. Since the last stage inverter outputs a signal that has a reversed logic as the input of the first stage inverter, the whole circuit can oscillate. The frequency of the ring oscillator is determined by the propagation delay of each inverter. We use two approaches to

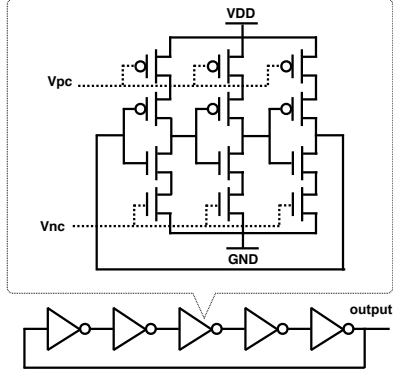

Figure 8: Ring oscillator circuit diagram.

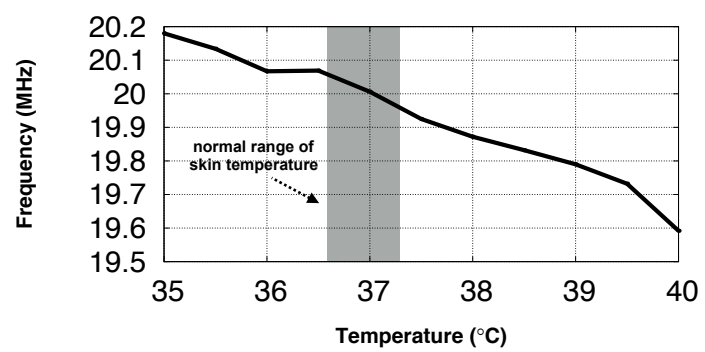

Figure 9: Ring oscillator frequency when temperature changes. The normal range of skin temperatures is fairly tight (typically between $36.6^{\circ} \mathrm{C}$ and $37.2^{\circ} \mathrm{C}$ ).

control the propagation delay of each stage. First, we use a voltage controlled inverter where we adjust the gate voltage $\left(V_{n c}\right.$ and $V_{p c}$ ) of two PMOS and NMOS transistors in an inverter to control its propagation delay. Second, we use an $\mathrm{RC}$ circuit between the inverters to add additional delay. We simulate a $20 \mathrm{MHz}$ ring oscillator in HSPICE and see that we are able to achieve $20 \mathrm{MHz}$ by tuning the control voltage $V_{n c}$ and $V_{p c}$ and the RC parameters.

While attractive from a power perspective, a ring oscillator is typically not used in active radios because its frequency can vary a fair bit with temperature variations. In general, the frequency can vary by a few $\mathrm{MHz}$ if there is a significant temperature swing of more than a few tens of degrees (C). Such variation is typically going to be a showstopper for many radio designs.

However, a ring oscillator may still be suitable for FSBackscatter since it is specifically intended for on-body sensors. The normal range of skin temperatures is fairly tight (typically between $36.6^{\circ} \mathrm{C}-37.2^{\circ} \mathrm{C}$ ), and even sweating and physical exercise only induce small temperature changes of less than $1^{\circ} \mathrm{C}$ due to thermal regulation [10]. Figure 9 shows an HSPICE simulation of our ring oscillator design at temperatures around the human range. We use our HSPICE implementation of the $20 \mathrm{MHz}$ ring oscillator to measure the effect of such temperature shifts, and find that the frequency changes by roughly $69 \sim 210 \mathrm{kHz}$. We then modify the shifted frequency of FS-Backscatter by $20 \mathrm{MHz} \pm 250 \mathrm{kHz}$ to see its effect on the packet-level and bit-level decoders described earlier.

Figure 10(a) shows the effect on a packet-level decoder when leveraging a WiFi signal. When an FS-Backscatter tag experiences a frequency offset that is smaller than $100 \mathrm{kHz}$, we can achieve similar throughput as the one without any frequency offset. However, when the frequency offset is larger 


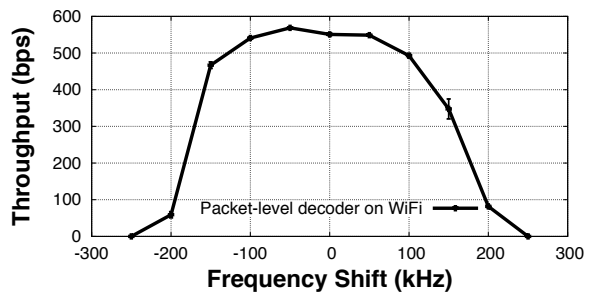

(a) Packet-level decoder on a WiFi signal.

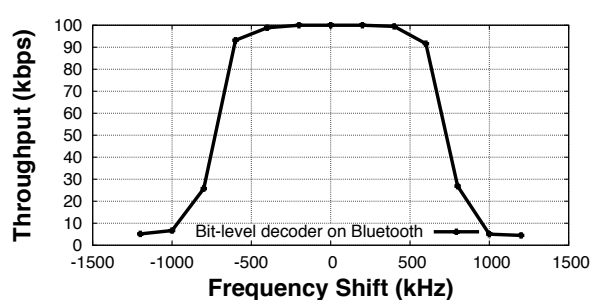

(b) Bit-level decoder on a Bluetooth signal.

Figure 10: Packet-level and bit-level decoder throughput when an FS-Backscatter tag experiences frequency offset.

than $150 \mathrm{kHz}$, FS-Backscatter throughput starts degrading. When the frequency offset is larger than $250 \mathrm{kHz}$, FS-Backscatter throughput degrades to zero. While not shown in the figure, we also see that BLE packet-level decoder is more robust to frequency shifts, and can tolerate roughly $450 \mathrm{kHz}$ frequency shift before the throughput degrades.

Note that even if the packet-level decoder does not work when the sensor is not attached to the body or when the temperature swing is large, we can still use the bit-level decoder that uses RSSI information. Figure 10(b) shows the effect on a bit-level decoder when leveraging a Bluetooth signal. Bit-level decoder is more robust to the frequency offset compared to the packet-level decoder. We observe that its throughput degrades only when the frequency offset is larger than $600 \mathrm{kHz}$. Since Bluetooth channels are $2 \mathrm{MHz}$ apart, a temperature-compensated decoder can listen on the appropriate channel where the backscattered signal is strongest.

\subsubsection{Reducing operating voltage}

Another optimization that we make is to reduce the voltage range in which the FS-Backscatter tag operates and thereby reduce power. Let us first look at the voltage needed for toggling an RF transistor. The minimum voltage needed for powering an ADG902 RF transistor is $V_{D D}=1.65 \mathrm{~V}$. However, it does not mean that we need to feed a $1.65 \mathrm{~V}$ signal into the gate of the transistor for opening and closing the gate. In fact, an ADG902 can be opened and closed by switching between $0.65 V_{D D}$ and $0.35 \mathrm{~V}$. As a result, instead of switching between $1.65 \mathrm{~V}$ and $0 \mathrm{~V}$, we can switch between $1.0725 \mathrm{~V}$ and $0.35 \mathrm{~V}$ to toggle the transistor. Such smaller operational voltage range will reduce the power consumed for toggling the RF transistor.

Similarly, we do not have to run the ring oscillator and the data modulator at high voltage either. Instead of running the whole system at $1.65 \mathrm{~V}$, we can operate these two subsystems at $0.8 \mathrm{~V}$. Then, we use a $0.3 \mathrm{~V}$ voltage shifter to move the $0.8 \mathrm{~V} / 0 \mathrm{~V}$ signal output by the modulator to $1.1 \mathrm{~V} / 0.3 \mathrm{~V}$, high

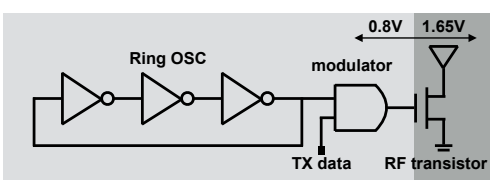

Figure 11: FS-Backscatter tag diagram.

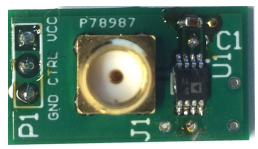

Figure 12: FS-Backscatter radio analog front end

enough for toggling the RF transistor. By operating the ring oscillator and modulator at $0.8 \mathrm{~V}$, we can significantly reduce the overall system power consumption. Our final tag design is shown in Figure 11 .

\section{IMPLEMENTATION}

In this section, we describe our implementation of FSBackscatter.

FS-Backscatter Tag: Our prototype of an FS-Backscatter tag is designed to be flexible in connecting different types of antennas to understand the effect of antenna gain. The backscatter analog front end that allows us to explore these design options is shown in Figure 12. We use an ADG902 transistor to tune and detune the antenna. The antenna is connected to the transistor via an SMA connector, which allows us to directly connect different types of antennas. For example, we connect to a VERT2450 and a TL-ANT2409A $2.4 \mathrm{GHz}$ antenna for reflecting $2.4 \mathrm{GHz}$ wireless signals in our implementation. Our flexibility comes at a cost, however, since we do not tune matching circuits to the specific antenna. Hence, we might expect some performance improvement in a more integrated version.

In addition to the above prototype, we also have a full simulation of FS-Backscatter in HSPICE, which allows us to evaluate the power and performance of our ring oscillator circuit and voltage rails optimizations. We use three voltage controlled inverters to implement the ring oscillator. The control voltages for PMOS and NMOS are $V_{p c}=0.1 \mathrm{~V}$ and $V_{n c}=0.75 \mathrm{~V}$ respectively. We add one RC circuit ( $R=1.008 \mathrm{~K}, C=1.84 \mathrm{pF})$ in the second stage of the ring oscillator to introduce additional delay. When we use $0.8 \mathrm{~V}$ to drive the PMOS and NMOS inside of the ring oscillator, we are able to obtain $20.006 \mathrm{MHz}$ oscillating frequency, accurate enough for modulating our information. Before feeding the $20 \mathrm{MHz}$ clock into the modulator, we put two additional inverters after the ring oscillator to shape the signal output by the ring oscillator.

Active transmitter and FS-Backscatter decoder: Our carrier transmitter and receiver implementations use standard radios with standard antenna configurations to keep the setup similar to what we can expect in a mobile scenario. The transmitter is simply a Bluetooth/BLE or WiFi transmitter that continuously broadcasts data in a specified channel. Our packet-level decoders are implemented on a commercial TI CC3200 WiFi receiver and TI CC2650 BLE receiver. Packet-level reception is designed to work on commercial 


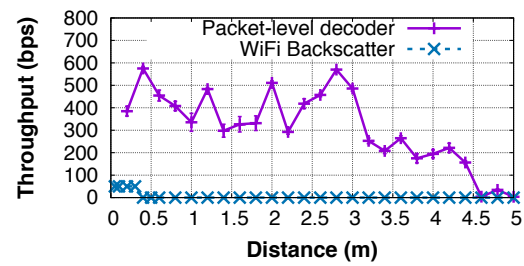

(a) Throughput when reflecting WiFi.

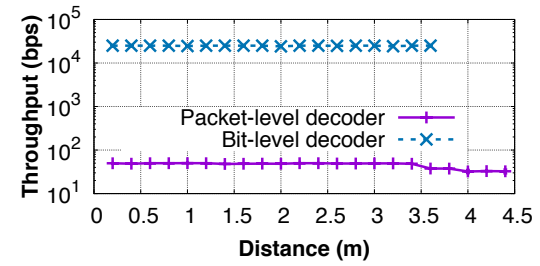

(b) Throughput when reflecting BLE.

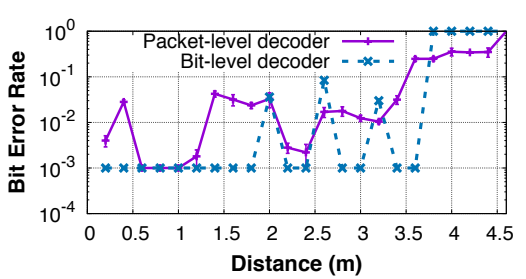

(c) BER when reflecting BLE.

Figure 13: FS-Backscatter throughput and BER across distance when leveraging WiFi and Bluetooth signals.

WiFi and BLE receivers without modification. Our bit-level decoder is implemented on a TI CC2541 BLE chipset which, in addition to the normal BLE mode, also supports a proprietary mode that bypasses the Bluetooth stack and allows us to directly access channel RSSI. While this API is not widely available on all BLE chipsets, we note that this mode is only needed at the receiver i.e. only one endpoint needs modification. So, one potential path to widespread use may be to have next-generation fitness bands or smartwatches swap BLE chipsets to use one with low-level channel access (or otherwise provide API access to the raw channel RSSI values) so that we can also use it as a high-rate backscatter receiver.

When we observe an incident WiFi signal on the $i$ th channel and a Bluetooth/BLE signal on the $j$ th channel, we configure CC3200 and CC2650/CC2541 to detect packets on the $i+n$th and $j+n$th channels where $n$ indicates the number of channels shifted by an FS-Backscatter tag. Signals detected by each radio are reported to the joint decoder for deciding the actual bit transmitted by a backscatter tag. CC3200, CC2650, and CC2541 have similar sensitivity $(-95 \mathrm{dBm})$ for detecting a backscattered signal.

WiFi Backscatter setup: Since the code for WiFi Backscatter is under license from UW to a licensee company, we reimplement this scheme using parameters provided in the paper. We use a $9 \mathrm{dBi}$ directional gain antenna at the tag in experiments where we compare against this scheme because WiFi Backscatter does not work with a monopole antenna (as described in \$2 . But in all other experiments, we use a standard 3dBi omni-directional antenna [2] for FS-Backscatter. The WiFi/Bluetooth transmitter and receivers are equipped with standard onboard chip or PCB antennas.

\section{EVALUATION}

We now turn to an evaluation of the various aspects of FSBackscatter.

\subsection{FS-Backscatter: Throughput and BER}

Our goals in this experiment are two-fold. First, we want to tease apart the benefits of shifting to a clean band, and leveraging structure of WiFi/Bluetooth packets. Packet-level decoding allows us to take advantage of both whereas bitlevel decoding only allows us to take advantage of the clean band. Second, we want to understand the differences in obtained throughput if we use the two types of decoding methods. Packet-level decoding gets one bit of information per packet, whereas bit-level decoding can go much faster.
In this experiment, we place a backscatter tag $1 \mathrm{~m}$ away from a CC $3200 \mathrm{WiFi} / \mathrm{CC} 2650 \mathrm{BLE}$ transmitter and then move the backscatter decoder away from the tag. We show two versions of this experiment - the first with a $9 \mathrm{dBi}$ directional antenna [5] on the tag to ensure that we obtain throughput numbers for WiFi Backscatter, and the second using a more standard $3 \mathrm{dBi}$ antenna [2]. We then evaluate the throughput for FS-Backscatter across distance for packet-level and bitlevel decoding in FS-Backscatter as well as WiFi Backscatter.

Figure 13(a) shows the results. Packet-level decoding generally gives us the highest range of $\sim 5 \mathrm{~m}$, demonstrating the benefits of leveraging both a clean band as well as signal structure. We get this range even when using a more typical monopole antenna, which shows that leveraging both benefits has huge implications on range and makes backscatter practical even in challenging environments. FS-Backscatter can achieve $4.8 \mathrm{~m}$ maximum operational distance, $16 \times$ longer than the WiFi backscatter system. In addition, the average throughput achieved is $627.7 \mathrm{bps}, 12.5 \times$ higher than WiFi Backscatter. These results clearly show the benefits of moving the backscattered signal into an adjacent clean spectrum rather than trying to separate WiFi signal variation from the backscatter modulated signal within the same band.

We turn to a comparison of packet-level decoding v.s. bitlevel decoding. We use a BLE transmitter, and show results for the two decoding schemes in Figure 13(b) When bit-level RSSI information is used for decoding, the maximum operational distance achieved is $3.6 \mathrm{~m}$ and the throughput increases to $50 \mathrm{kbps}, 79 \times$ higher than FS-Backscatter when packet-level RSSI is used because intra-packet RSSI detection allows us to detect the presence of reflected signal faster. The achieved maximum operational distance is slightly shorter because bit-level RSSI does not leverage the packet structure for decoding.

Figure 13(c) shows the bit error rate (BER) of FS-Backscatter across distance. We use the same experimental setting as Figure 13(b). FS-Backscatter with bit-level decoder can achieve $10^{-3}$ BER at $3.6 \mathrm{~m}$ with $50 \mathrm{kbps}$ data rate and packet-level decoder can achieve $10^{-2}$ BER at $3.2 \mathrm{~m}$. When the FS-Backscatter tag is further, bit-level decoder BER increases to one sharply while packet-level decoder BER increases gradually. Such difference comes from the fact that packet-level decoder can leverage the structure of a packet for detecting the reflected signal. As a result, it is more tolerant to the degradation of reflected signal strength. 


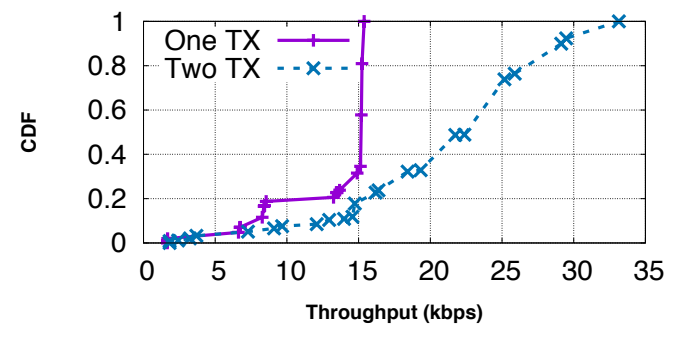

(a) Leveraging two active transmitters.

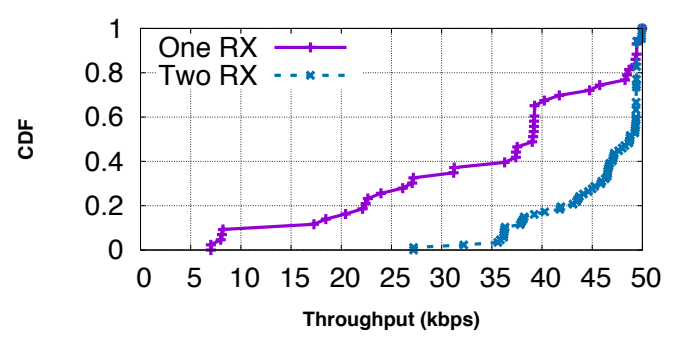

(b) Leveraging two active receivers.

Figure 14: FS-Backscatter throughput benefit when leveraging multiple active transmitters and receivers.

\subsection{Multiple Carriers and Receivers}

Let us now look at the benefits of leveraging multiple carrier signals for carrying backscattered information and multiple receivers for joint decoding. This set of experiments considers scenarios where we might have three or more radios on a phone, wristband, and tablet, and where multiple transmitters or receivers may be leveraged.

Leveraging multiple carriers First, we investigate the benefit of multiple carriers where two Bluetooth signals are simultaneously leveraged by FS-Backscatter. We deploy two Bluetooth transmitters $0.2 \mathrm{~m}$ away from each other and a FSBackscatter tag in five locations in the department building, run a 2-minute experiment at each location, and compute throughput once every 10 seconds. Figure 14(a) shows the cumulative throughput of FS-Backscatter. When we only leverage a single Bluetooth signal, median throughput of $15.1 \mathrm{kbps}$ is achieved. However, FS-Backscatter is able to achieve $22.3 \mathrm{kbps}$ median throughput when leveraging both transmitters, $1.47 \times$ higher than leveraging a single Bluetooth signal. The throughput improvement is for reasons described in $\$ 3.1 .4$ - since Bluetooth transmitters are limited to a maximum output power of $0 \mathrm{dBm}$, two transmitters naturally increases the signal strength at the decoder.

Leveraging multiple receivers In our second experiment, we look at the case where two receivers are leveraged for joint decoding. We use two Bluetooth receivers $0.2 \mathrm{~m}$ away from each other, each of which is configured to decode by using bit-level RSSI information. We measure the cumulative throughput and show the results in Figure 14(b) When a single Bluetooth receiver is used, we achieve $39.1 \mathrm{kbps}$ median throughput. When we jointly decode using two Bluetooth receivers, we can achieve $48.7 \mathrm{kbps}$ throughput, $1.24 \times$ higher than the single receiver case. We can achieve such through-

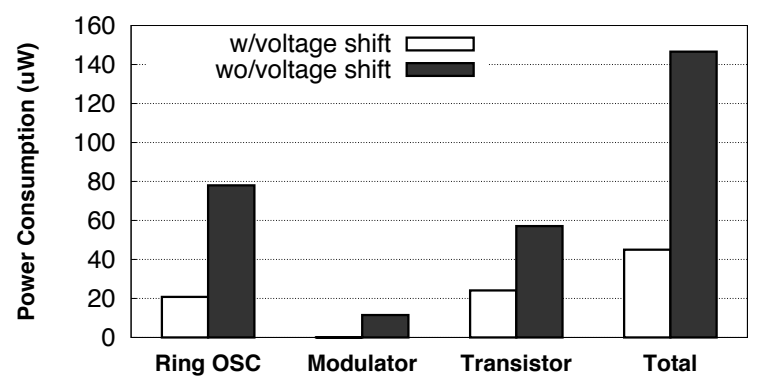

Figure 15: Benchmarking the power consumption of a FSBackscatter tag.

put improvement because the reflected signal at one receiver can be strong while the reflected signal at the other is weak. In these cases, joint decoding is helpful and improves SNR.

\subsection{Power consumption}

Let us now look at the power consumption of an FS-Backscatter tag. We provide a breakdown of power for each component (ring oscillator, modulator, RF transistor), as well with and without DC voltage shifting. The results are shown in Figure 15

We first look at the tag power consumption without DC voltage shifting where the whole system operates at $1.65 \mathrm{~V}$, which is the minimum voltage required for toggling an ADG902 RF transistor. The three hardware components of an FSBackscatter tag: ring oscillator, data modulator, and RF transistor consume $78 \mu \mathrm{W}, 11.5 \mu \mathrm{W}$, and $57.1 \mu \mathrm{W}$ respectively when transmitting at $50 \mathrm{kbps}$ and the overall tag power consumption is $146.6 \mu \mathrm{W}$.

We reduce the tag power consumption by configuring the ring oscillator and data modulator to operate at lower voltage $(0.8 \mathrm{~V})$ and shift the signal voltage output by the data modulator before feeding into the RF transistor. In this case, the ring oscillator, data modulator, and RF transistor consume $20.8 \mu \mathrm{W}, 0.1 \mu \mathrm{W}$, and $24.1 \mu \mathrm{W}$ respectively with DC voltage shifting. The overall tag power consumption is $45 \mu \mathrm{W}, 3.25 \times$ lower than the case without DC voltage shifting. As shown, the major power reduction comes from the ring oscillator, which consumes $3.75 \times$ less power when operating at a lower voltage.

\subsection{FS-Backscatter vs BLE/Zigbee}

In this section, we compare the performance of FS-Backscatter against low-power active radios such as BLE and Zigbee. Low-power radios for wearable devices need to be compared along two axes. The first is bits/joule i.e. how many bits can be transmitted for a fixed amount of energy. This gives a measure of how much data can be transferred via a particular radio given an energy budget. However, more powerful radios with higher bitrates will generally have higher efficiency in bits per joule, but will also consume more power in active mode. To account for this effect, another metric that is useful is peak power draw of the radio. Higher peak power draw implies worse lifetime from batteries, since battery decay curves are linked to not just the average power draw but also the peak power draw [16] [15] [7]. It also means that 
Table 2: FS-Backscatter energy efficiency. Pkt refers to packet-level decoding, and Bit refers to bit-level decoding.

\begin{tabular}{|l|c|c|}
\hline & Bits/ $\mu \mathrm{J}$ & Peak Power \\
\hline BLE(CC2650) & 54.6 & $18.3 \mathrm{~mW}(0 \mathrm{dBm})$ \\
\hline ZigBee(CC2630) & 13.7 & $18.3 \mathrm{~mW}(0 \mathrm{dBm})$ \\
\hline FS-Backscatter (Pkt-WiFi) & 25.5 & $45 \mu \mathrm{W}$ \\
\hline FS-Backscatter (Pkt-BLE) & 2.2 & $45 \mu \mathrm{W}$ \\
\hline FS-Backscatter (Bit) & 1100 & $45 \mu \mathrm{W}$ \\
\hline
\end{tabular}

tags would need more complex batteries with built-in power management circuits to be able to sustain the burst during active mode. In addition, higher peak power also means that operating on harvested power is unlikely since additional voltage boosting and energy buffering circuits increase quiescent power draw.

Table 2 shows the peak power consumption and bits per $\mu \mathrm{J}$ of a CC2650 BLE radio, a CC2630 ZigBee radio, and FSBackscatter operating in three modes. FS-Backscatter has three orders of magnitude smaller peak power consumption compared to BLE and ZigBee. Therefore, FS-Backscatter is beneficial when we design a system that requires small peak power consumption, for example, in energy harvesting-based tags. When leveraging packet-level decoding, FS-Backscatter has smaller bits per $\mu \mathrm{J}$ compared to BLE and ZigBee because its data rate is slow. However, when operating in bit-level decoding mode, FS-Backscatter energy efficiency significantly improves and can achieve 1100bits/ $\mu \mathrm{J}, 20.3 \times$ higher than BLE.

\subsection{Mobile and static deployment}

We now look at the overall system performance in an onbody sensing scenario where we place a BLE transmitter in the pocket, a BLE receiver on the wrist, and the FS-Backscatter tag on the chest. This scenario corresponds to a scenario where a user has a phone and smartwatch, and wears an onbody sensor. The FS-Backscatter sensor tag transmits data at $50 \mathrm{kbps}$, and a wristband receives and decodes the reflected signal using bit-level decoding. We look at a static case where the user is static for 10 minutes, and a mobile case where the user moves around for 10 minutes. We plot CDFs of the throughput numbers taken for each 10 second interval.

Figure 16 shows the results. We are able to achieve $48.7 \mathrm{kbps}$ and $21 \mathrm{kbps}$ median throughput when the person is static and mobile respectively. These numbers are very encouraging since many on-body sensors generate data rates far lower than this number. For example, a 3-axis accelerometer [1] sampled at $100 \mathrm{~Hz}$ has a data communication rate requirement of $4.8 \mathrm{kbps}$, whereas a ECG electrode [6] sampled at $250 \mathrm{~Hz}$ has data rate requirements of $2 \mathrm{kbps}$.

Between the static and mobile cases, we observe higher and more stable FS-Backscatter throughput in static deployment, as we might expect. In contrast, FS-Backscatter throughput is lower in the mobile deployment because body movement leads to significant channel variations and degrades throughput. However, the throughput in the mobile case is also quite promising, and worst case throughput is already more than 10kbps. This means that FS-Backscatter should generally

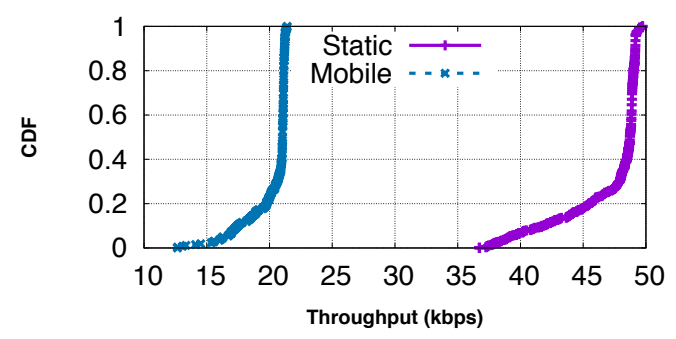

Figure 16: FS-backscatter throughput in static and mobile deployment.

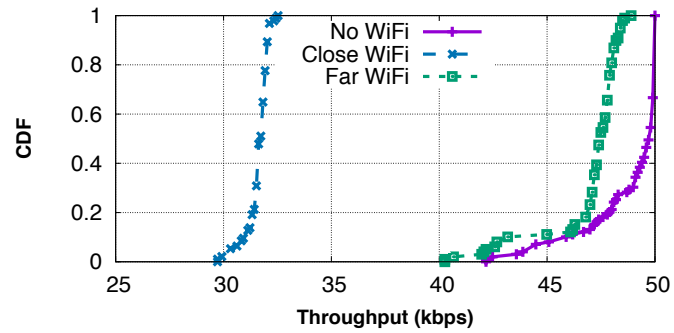

(a) FS-Backscatter throughput when WiFi interference is present.

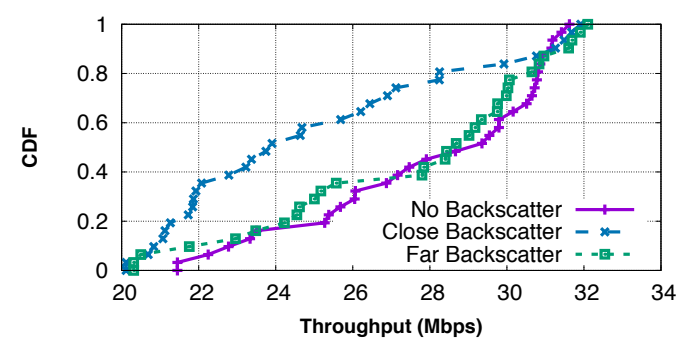

(b) WiFi throughput when backscatter interference is present.

Figure 17: Mutual interference between FS-Backscatter and WiFi.

be able to provide a continuous communication link from an on-body sensor to a phone/smartwatch combination.

\subsection{Mutual Interference}

One potential issue that we have not touched upon so far is how FS-Backscatter might interfere or be interfered by active radio traffic. Understanding mutual interference is important because FS-Backscatter operates in the $2.4 \mathrm{GHz}$ ISM band where spectrum occupancy is high. To answer this question, we first look at the interference by a WiFi radio on FSBackscatter when both operate on the same channel. We deploy both a BLE transmitter and a FS-Backscatter decoder $1 \mathrm{~m}$ away from an FS-Backscatter tag. We then adjust the distance of a WiFi interferer to understand how WiFi interferes with the FS-Backscatter data transmission. We use the bit-level decoder in this experiment since this is most likely to be impacted by cross-traffic.

Figure 17(a) shows that FS-Backscatter can achieve 49.7kbps median throughput when the WiFi interferer is not present. However, we observe that throughput degrades to $31.7 \mathrm{kbps}$ and $47.4 \mathrm{kbps}$ when the WiFi interferer is $10 \mathrm{~m}$ and $15 \mathrm{~m}$ from the FS-Backscatter decoder. This is because the power of 


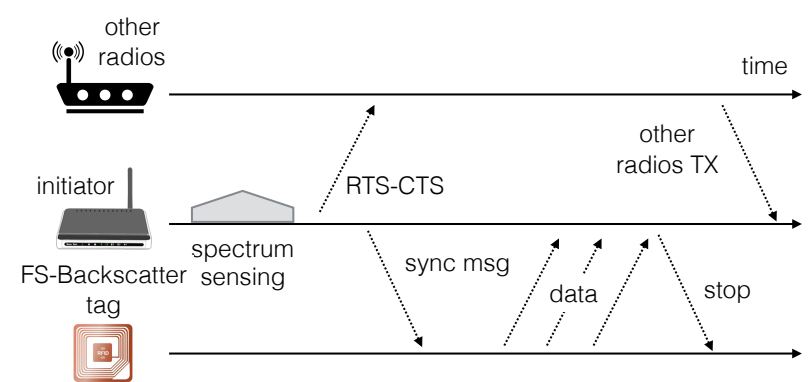

Figure 18: One MAC protocol that can be run by the backscatter communication initiator.

the backscattered signal is only around $-80 \mathrm{dBm}$, whereas the power of the WiFi interferer is at least $20 \mathrm{~dB}$ higher at close range. When the WiFi transmitter is $15 \mathrm{~m}$ away, FSBackscatter is able to achieve $47.4 \mathrm{kbps}$ throughput, close to the case when the WiFi interferer is not present. When WiFi transmitter is closer than $10 \mathrm{~m}$, FS-Backscatter throughput degrades to zero because $\mathrm{WiFi}$ interference is too strong.

Let us now look how FS-Backscatter interferes on an ongoing WiFi transmission. In this experiment, we deploy a WiFi transmitter $5 \mathrm{~m}$ away from a WiFi receiver. Figure $17(\mathrm{~b})$ shows that WiFi is able to achieve 29Mbps when FS-Backscatter is not present. When a FS-Backscatter tag is $0.2 \mathrm{~m}$ from the WiFi receiver, the median WiFi throughput degrades to $23.9 \mathrm{Mbps}, 1.21 \times$ smaller. When the FS-Backscatter tag is $1 \mathrm{~m}$ away, we observe $28.7 \mathrm{Mbps}$ median WiFi throughput, close to the case when FS-Backscatter is not present. Therefore, FS-Backscatter has only a relatively small interference range, and even then does not seem to have a substantial effect on WiFi throughput.

\section{DISCUSSION}

MAC protocol: One problem that we have not discussed is how a FS-Backscatter tag co-exists with other radios operating in the $2.4 \mathrm{GHz}$ band, such as WiFi and Bluetooth. We cannot implement CSMA-like MAC control mechanisms on a FS-Backscatter tag because spectrum sensing consumes several milli-Watts of power which makes it infeasible on a low-power tag. But it may be possible to shift this functionality to the backscatter communication initiator, which is less power constrained. The initiator does this in two steps. First, the initiator senses the availability of the wireless spectrum. It needs to check at least two adjacent channels because one channel will be used by the initiator and the other will be used by an FS-Backscatter tag. Then, the initiator reserves both channels, and informs the FS-Backscatter tag to start backscatter communication. Figure 18 shows the timing diagram of a MAC protocol that can be run by the initiator. Once the available spectrum is identified, the initiator sends an RTS-CTS message to reserve both channels. A caveat is that the initiator is only able to reserve both channels for a limited amount of time. Therefore, it has to inform the FS-Backscatter tag about the amount of data that can be backscattered during the window.

Interscatter v.s. FS-Backscatter: A concurrently published research result that also addresses the problem of en- abling backscatter on wearables and implantables while relying solely on commodity radios is Interscatter [26]. Interscatter and FS-Backscatter have some differences but also complement each other in several ways. Unlike Interscatter, which uses a Bluetooth transmitter and WiFi receiver, FSBackscatter enables backscatter with multiple types of active radios (WiFi-to-WiFi/Bluetooth, Bluetooth-to-WiFi/Bluetooth) as long as these radios can transmit and receive at different channels. In addition, unlike Interscatter, FS-Backscatter does not need to generate the WiFi or Bluetooth baseband signal and therefore should incur lower power consumption. On the one hand, the single side band modulation technique used by Interscatter is an interesting innovation that can be used by FS-Backscatter for improving its backscatter spectrum efficiency. FS-Backscatter and Interscatter also differ in their choice of oscillators - FS-Backscatter uses a ring oscillator which is lower power but has more temperatureinduced variations, whereas Interscatter uses a Phase Lock Loop (PLL), which has higher stability but higher power consumption [22]. The appropriate choice depends on the choice of application and the temperature variability.

Reducing tag power consumption: One of the design options that we made in FS-Backscatter was to shift by $20 \mathrm{MHz}$ because our target was to backscatter both WiFi and Bluetooth signals. However, if we are focused solely on shifting a Bluetooth carrier to a Bluetooth receiver in the adjacent band, the amount of frequency shift needed can be reduced dramatically to a $2-4 \mathrm{MHz}$. This choice can greatly reduce power consumption of the oscillator, and therefore the entire tag, and may be a preferred option in cases where a lower power tag is necessary.

\section{RELATED WORK}

There has been a lot of interest and activity in the area of backscatter-based communication and sensing in recent years $[20,23,24,25,35,41,48,53,54,55,56]$. The interest has been spurred by the booming industry for embedding tiny sensors in virtually anything that we wear, touch, use or even ingest, ranging from the Internet of Things, on-body and implantable sensors, wearables, mobile devices, urban sensing, and others [8, 19, 36, 47, 51].

In particular, our work is inspired by recent progress on enabling backscatter with commodity radios or with some infrastructure support. Among the earliest efforts at approaching the problem in this manner is Ambient Backscatter [34] and WiFi backscatter [30]. More recently, there have been interesting infrastructure-assisted approaches such as BLEBackscatter [13] and BackFi [9]. We have discussed these methods extensively in \$2, and will not go into the details here. These are terrific ideas but they do have their limitations either in terms of robustness or practicality in the mobile environment. We build on these ideas and look at how to make backscatter practical for on-body devices. We also note that prior work does not look at the possibility of leveraging multiple incident signals, which we can take advantage of in FS-Backscatter. 
FS-Backscatter is also inspired by previous work on interference cancellation. Recent work has looked at this problem in the context of full-duplex radios[11, 27, 37, 42]. However, these efforts require additional hardware components that are not present on many existing commercial radios. Other recent work use signal processing techniques over the analog signal to minimize interference [17, 18, 21, 29, 33]. However, such analog signals are not available on many existing commercial radios. FS-Backscatter is designed to work on commercial radios and their constraints, and uses frequency shifting rather than interference cancelation.

FS-Backscatter tag is also inspired by previous work on RFID tag ASIC designs [38, 44, 50]. The main difference between an FS-Backscatter tag and an RFID tag is that FSBackscatter requires a higher speed local clock for shifting the incident carrier signal. To achieve this, we leverage ring oscillators designs [14, 40, 43] and tune the circuit to enable $20 \mathrm{MHz}$ oscillating frequency while only consuming $\sim 20 \mu \mathrm{W}$ of power.

\section{CONCLUSION}

In summary, we discuss the design of FS-Backscatter, a system that enables backscatter communication between onbody sensor tags and commercial WiFi and Bluetooth radios. The key idea of FS-Backscatter is that we can reduce carrier signal interference by shifting the backscattered signal to a clean band that does not overlap with the carrier. We demonstrate that a $20 \mathrm{MHz}$ frequency shift is enough for enabling an FS-Backscatter tag to communicate with commercial WiFi and Bluetooth radios. Such frequency shift does not come with high power consumption at the tag side because we leverage a ring oscillator circuit to design a FSBackscatter tag that only consumes $45 \mu \mathrm{W}$. Our empirical evaluation shows that an FS-Backscatter tag is able to communicate with commercial $\mathrm{WiFi}$ and Bluetooth radios up to $4.8 \mathrm{~m}$ and achieve $50 \mathrm{kbps}$ data rate. We believe that FSBackscatter paves the way toward enabling practical deployment of backscatter-based low power on-body sensor tags.

\section{Acknowledgements}

We thank the shepherd Suman Banerjee and the anonymous reviewers for their insightful comments. This research was partially funded by NSF grants CNS-1218586 and CNS-1217606.

\section{REFERENCES}

[1] Adx1362 mems accelerometer.

[2] Ettus research vert2450 antenna.

[3] Nxp 74hc1g00 nand gate.

[4] Ti cc2541.

[5] Tp-link tl-ant2409a antenna.

[6] Zephyr bioharness.

[7] A. Badam, R. Chandra, J. Dutra, A. Ferrese, S. Hodges, P. Hu, J. Meinershagen, T. Moscibroda, B. Priyantha, and E. Skiani. Software defined batteries. In SOSP, 2015.
[8] S. Bandyopadhyay, P. P. Mercier, A. C. Lysaght, K. M. Stankovic, and A. P. Chandrakasan. A $1.1 \mathrm{nw}$ energy-harvesting system with the pw quiescent power for next-generation implants. JSSC, 2014.

[9] D. Bharadia, K. R. Joshi, M. Kotaru, and S. Katti. Backfi: High throughput wifi backscatter. In SIGCOMM, 2015.

[10] W. Bierman. The temperature of the skin surface. Journal of the American Medical Association, 1936.

[11] J. I. Choi, M. Jain, K. Srinivasan, P. Levis, and S. Katti. Achieving single channel, full duplex wireless communication. In Mobicom, 2010.

[12] F. C. Commission. Fcc part 15.247.

[13] J. F. Ensworth and M. S. Reynolds. Every smart phone is a backscatter reader: Modulated backscatter compatibility with bluetooth 4.0 low energy (ble) devices. In RFID. IEEE, 2015.

[14] S. Farzeen, G. Ren, and C. Chen. An ultra-low power ring oscillator for passive uhf rfid transponders. In Circuits and Systems (MWSCAS), 2010 53rd IEEE International Midwest Symposium on, pages 558-561. IEEE, 2010.

[15] L. M. Feeney, C. Rohner, P. Gunningberg, A. Lindgren, and L. Andersson. How do the dynamics of battery discharge affect sensor lifetime? In 11th Annual Conference on Wireless On-demand Network Systems and Services, 2014.

[16] K. Furset and P. Hoffman. High pulse drain impact on cr2032 coin cell battery capacity.

[17] S. Gollakota, F. Adib, D. Katabi, and S. Seshan. Clearing the rf smog: making $802.11 \mathrm{n}$ robust to cross-technology interference. SIGCOMM, 2011.

[18] S. Gollakota and D. Katabi. Zigzag decoding: combating hidden terminals in wireless networks. In SIGCOMM, 2008.

[19] S. Gollakota, M. S. Reynolds, J. R. Smith, and D. J. Wetherall. The emergence of rf-powered computing. Computer, 47(1):32-39, 2014.

[20] J. Gummeson, P. Zhang, and D. Ganesan. Flit: a bulk transmission protocol for rfid-scale sensors. In MobiSys, 2012.

[21] D. Halperin, T. Anderson, and D. Wetherall. Taking the sting out of carrier sense: interference cancellation for wireless lans. In Mobicom, 2008.

[22] M.-t. Hsieh and G. E. Sobelman. Comparison of lc and ring vcos for plls in a $90 \mathrm{~nm}$ digital cmos process. Proceedings, Int. SoC, 2006.

[23] P. Hu, P. Zhang, and D. Ganesan. Leveraging interleaved signal edges for concurrent backscatter. In HotWireless, 2014.

[24] P. Hu, P. Zhang, and D. Ganesan. Laissez-faire: Fully asymmetric backscatter communication. In SIGCOMM, 2015.

[25] P. Hu, P. Zhang, M. Rostami, and D. Ganesan. Braidio: An integrated active-passive radio for mobile devices with asymmetric energy budgets. In SIGCOMM, 2016. 
[26] V. Iyer, V. Talla, B. Kellogg, S. Gollakota, Shyam, and Josh. Interscatter: Towards internet connectivity for medical implants. In SIGCOMM, 2016.

[27] M. Jain, J. I. Choi, T. Kim, D. Bharadia, S. Seth, K. Srinivasan, P. Levis, S. Katti, and P. Sinha. Practical, real-time, full duplex wireless. In Mobicom, 2011.

[28] P. Kamalinejad, K. Keikhosravy, R. Molavi, S. Mirabbasi, and V. Leung. An ultra-low-power cmos voltage-controlled ring oscillator for passive rfid tags. In 12th International New Circuits and Systems Conference, 2014.

[29] S. Katti, H. Rahul, W. Hu, D. Katabi, M. Médard, and J. Crowcroft. Xors in the air: practical wireless network coding. In SIGCOMM, 2006.

[30] B. Kellogg, A. Parks, S. Gollakota, J. R. Smith, and D. Wetherall. Wi-fi backscatter: internet connectivity for rf-powered devices. In SIGCOMM, 2014.

[31] B. Kellogg, V. Talla, S. Gollakota, and J. R. Smith. Passive wi-fi: bringing low power to wi-fi transmissions. In NSDI, 2016.

[32] K. K. Lee, K. Granhaug, and N. Andersen. A study of low-power crystal oscillator design. In NORCHIP, 2013, pages 1-4. IEEE, 2013.

[33] K. C.-J. Lin, N. Kushman, and D. Katabi. Ziptx: Harnessing partial packets in 802.11 networks. In Mobicom, 2008.

[34] V. Liu, A. Parks, V. Talla, S. Gollakota, D. Wetherall, and J. R. Smith. Ambient backscatter: wireless communication out of thin air. In SIGCOMM, 2013.

[35] V. Liu, V. Talla, and S. Gollakota. Enabling instantaneous feedback with full-duplex backscatter. In Mobicom, 2014.

[36] P. P. Mercier, A. C. Lysaght, S. Bandyopadhyay, A. P. Chandrakasan, and K. M. Stankovic. Energy extraction from the biologic battery in the inner ear. Nature biotechnology, 30(12):1240-1243, 2012.

[37] P. V. Nikitin and K. Rao. Antennas and propagation in uhf rfid systems. challenge, 22:23, 2008.

[38] P. V. Nikitin, K. S. Rao, S. F. Lam, V. Pillai, R. Martinez, and H. Heinrich. Power reflection coefficient analysis for complex impedances in rfid tag design. IEEE Transactions on Microwave Theory and Techniques, 53(9):2721-2725, 2005.

[39] A. Pantelopoulos and N. G. Bourbakis. A survey on wearable sensor-based systems for health monitoring and prognosis. Systems, Man, and Cybernetics, Part C: Applications and Reviews, IEEE Transactions on, 40(1):1-12, 2010.

[40] S. Park, C. Min, and S. Cho. A 95nw ring oscillator-based temperature sensor for rfid tags in $0.13 \mu \mathrm{m}$ cmos. In Circuits and Systems, 2009. ISCAS 2009. IEEE International Symposium on, pages 1153-1156. IEEE, 2009.
[41] A. N. Parks, A. Liu, S. Gollakota, and J. R. Smith. Turbocharging ambient backscatter communication. In SIGCOMM, 2014.

[42] D. M. Pozar. Microwave engineering. John Wiley \& Sons, 2009.

[43] G. Qu and C.-E. Yin. Temperature-aware cooperative ring oscillator puf. In Hardware-Oriented Security and Trust, 2009. HOST'09. IEEE International Workshop on, pages 36-42. IEEE, 2009.

[44] K. S. Rao, P. V. Nikitin, and S. F. Lam. Impedance matching concepts in rfid transponder design. In Automatic Identification Advanced Technologies, 2005. Fourth IEEE Workshop on, pages 39-42. IEEE, 2005.

[45] A. P. Sample, D. J. Yeager, P. S. Powledge, A. V. Mamishev, and J. R. Smith. Design of an rfid-based battery-free programmable sensing platform. Instrumentation and Measurement, IEEE Transactions on, 57(11):2608-2615, 2008.

[46] F. Song, J. Yin, H. Liao, and R. Huang. Ultra-low-power clock generation circuit for epc standard uhf rfid transponders. Electronics Letters, 44(3):199-201, 2008.

[47] V. Talla, B. Kellogg, B. Ransford, S. Naderiparizi, S. Gollakota, and J. R. Smith. Powering the next billion devices with wi-fi. In CoNext, 2015.

[48] J. Wang, H. Hassanieh, D. Katabi, and P. Indyk. Efficient and reliable low-power backscatter networks. In SIGCOMM, 2012.

[49] D. Yeager, F. Zhang, A. Zarrasvand, N. T. George, T. Daniel, and B. P. Otis. A 9 a, addressable gen2 sensor tag for biosignal acquisition. Solid-State Circuits, IEEE Journal of, 45(10):2198-2209, 2010.

[50] M. Yip, R. Jin, H. H. Nakajima, K. M. Stankovic, and A. P. Chandrakasan. A fully-implantable cochlear implant soc with piezoelectric middle-ear sensor and arbitrary waveform neural stimulation. JSSC, 2015.

[51] P. Zappi, C. Lombriser, T. Stiefmeier, E. Farella, D. Roggen, L. Benini, and G. Tröster. Activity recognition from on-body sensors: accuracy-power trade-off by dynamic sensor selection. In Wireless sensor networks, pages 17-33. Springer, 2008.

[52] P. Zhang and D. Ganesan. Enabling bit-by-bit backscatter communication in severe energy harvesting environments. NSDI, 2014.

[53] P. Zhang, D. Ganesan, and B. Lu. Quarkos: Pushing the operating limits of micro-powered sensors. In HotOS, 2013.

[54] P. Zhang, J. Gummeson, and D. Ganesan. Blink: A high throughput link layer for backscatter communication. In MobiSys, 2012.

[55] P. Zhang, P. Hu, V. Pasikanti, and D. Ganesan. Ekhonet: high speed ultra low-power backscatter for next generation sensors. In Mobicom, 2014. 